December 2011. Hospital records were reviewed. Vital signs, apnea and seizure events, need for respiratory support, infection rates, amount of feedings, gastric residuals were investigated. Descriptive statistics and one way Anova test were applied.

Results Seventy ROP examinations in 34 infants were included. Mean birth weight was $1157 \pm 256(700-1945)$ g, mean gestational age was $28 \pm 1.6(25-32)$ weeks. Median duration of mechanical support was $15.1 \pm 12(0-50)$ days. Mean heart rate, respiratory rate and oxygen saturation were similar 24 hours before and after examination. There were no significant differences in apnea event and seizures. Gastric distention was seen in three babies, suspected necrotising enterocolitis in one infant.

Conclusions There were no demostrable systemic effects associated with ROP examination although infants seemed to be somewhat tired. Low incidence of severe systemic side effects may be associated with fingertip pressure on lacrimal duct and reducing the amount of feedings just before and after the examination.

\section{THE COMORBIDITY OF CHRONIC PAIN AND INSOMNIA IN A COMMUNITY ADOLESCENT SAMPLE: PREVALENCE AND ASSOCIATION WITH SOCIODEMOGRAPHIC AND PSYCHOSOCIAL FACTORS}

doi:10.1136/archdischild-2012-302724.1611

'WS Wong, ${ }^{1} \mathrm{~N}$ Siu, ${ }^{2} \mathrm{C}$ Wong. ${ }^{1}$ Department of Psychological Studies, The Hong Kong Institute of Education; ${ }^{2}$ Department of Applied Social Studies, City University of Hong Kong, Hong Kong, Hong Kong S.A.R.

Background and Aims The comorbidity of chronic pain and insomnia has received increasing research attention in Western clinical pediatric populations; yet, little is known about its sociodemographic and psychological correlates in non-Western community pediatric populations. This study aimed to examine the prevalence of comorbid chronic pain and insomnia and its associated factors in a community sample of Chinese adolescents.

Methods A total of 1,518 adolescents aged from 11-19 years participated in this school-based study. Apart from sociodemographic background, participants were assessed on chronic pain, insomnia, depression, perceived stress, and social support. Prevalence of cooccurrence of chronic pain and insomnia was determined. Subjects with single symptom were compared with those with symptom cooccurrence on pain characteristics and sleep patterns. Multiple regression model evaluated factors associated with symptom comorbidity.

Results The prevalence of comorbid chronic pain and insomnia was $19.1 \%$ (95\% CI: 16.9, 21.4). Fully adjusted stepwise regression analyses identified being female, more depressive symptoms, and higher perceived stress to be significantly associated with comorbid symptoms. Adolescents with both symptoms reported significantly more pain sites, higher worst pain, and higher pain-associated interference than those reported chronic pain only. Subjects with comorbid symptoms also had poorer subjective sleep quality, greater sleep disturbances, and more daytime dysfunction than those reported insomnia only.

Conclusions Our data offered preliminary evidence that comorbid chronic pain and insomnia occurred among about one-fifth in the present sample of Chinese community adolescents.

\section{PREMEDICATION FOR NEONATAL INTUBATION: CURRENT PRACTICE IN SAUDI ARABIA}

doi:10.1136/archdischild-2012-302724.1612

${ }^{1,2} \mathrm{R}$ Mosalli, ${ }^{3} \mathrm{~K}$ Al Faleh, ${ }^{4} \mathrm{~B}$ Paes. ${ }^{1} \mathrm{Umm}$ Al Qura University, Mecca; ${ }^{2}$ Department of Pediatrics, International Medical Center, Jeddah; ${ }^{3}$ Department of Pediatrics (Neonatal Division), King Saud University, Riyadh, Saudi Arabia; ${ }^{4}$ Department of Pediatrics (Neonatal Division), McMaster University, Hamilton, ON, Canada
Objective Despite strong evidence of the benefits of rapid sequence intubation in neonates, it is still infrequently utilized in neonatal intensive care units (NICU), contributing to avoidable pain and secondary procedure-related physiological disturbances.

Aim of the study was to assess the practice of premedication, regimens commonly used before elective endotracheal intubation and neonatologists attitudes regarding this intervention in institutions across Saudi Arabia and to develop evidence based recommendations.

Methods A web based, structured questionnaire was constructed to assess the use of premedication for elective endotracheal intubation and determine barriers to the procedure. The questionnaire was distributed via e-mail to neonatal specialists and consultants of 10 NICUs.

Results 68 (85\%) of the clinicians responded to the survey. Most respondents were NICU consultants. Although 48 of the 68 responding physicians $(70 \%)$ believed it was essential to routinely use premedication for all elective intubations, only 28 (41\%) implemented this strategy. Fear of potential side effects was the most frequently cited reason for avoiding premedication. Treatment regimens varied widely among respondents.

Conclusion Rates of premedication prior to non-emergent intubation in neonates are suboptimal. Flawed information and lack of unified unit policy hampered effective implementation. Development of evidence based guideline may support country-wide adoption of this practice.

\section{MANAGEMENT OF VASO-OCCLUSIVE CRISIS WITH PATIENT CONTROLLED ANALGESIA}

doi:10.1136/archdischild-2012-302724.1613

1 JL Wayenberg, ${ }^{2} \mathrm{~F}$ De Pooter, ${ }^{3} \mathrm{~S}$ Redant, ${ }^{1} \mathrm{~J}$ Fontaine, ${ }^{4} \mathrm{~L}$ Dedeken, ${ }^{4} \mathrm{~S}$ Huybrechts, ${ }^{4} \mathrm{PO}$ Le, ${ }^{4} \mathrm{C}$ Heijmans, ${ }^{4} \mathrm{M}$ Ngalula Mujinga, ${ }^{4} \mathrm{~A}$ Ferster. ${ }^{1}$ Pain Ressource Unit, Department of Pediatrics; ${ }^{2}$ Anesthesiology; ${ }^{3}$ Emergency Unit, Department of Pediatrics; ${ }^{4}$ Hemato-Oncology Unit, Department of Pediatrics, Hopital Universitaire des Enfants Reine Fabiola (Université Libre de Bruxelles), Brussels, Belgium

Pain resulting from sickle-cell vaso-occlusive crisis (VOC) is often severe, prolonged and difficult to alleviate. Guidelines based on scientific evidence are lacking. In order to evaluate the effectiveness of our treatment protocol, we performed a population-based retrospective observational study on HbSS sickle-cell patients $(n=22)$ admitted for severe VOC $(n=48)$ during a 30 -months period and managed with patient controlled analgesia (PCA).

Median $\left(10^{\text {th }}-90^{\text {th }}\right.$ percentiles) visual analogical pain scale (VAS) at admission was $9.5(7-10)$. Patient received $0.3 \mathrm{mg} / \mathrm{kg}(0.1-0.4)$ intravenous morphine at admission, then PCA was started with the following settings: continuous rate: $20 \mu \mathrm{g} / \mathrm{kg} / \mathrm{h}(10-25)$, bolus: $25 \mu \mathrm{g} / \mathrm{kg}(21-32)$, and 1.8 bolus allowed/hour (1.7-2.8). Six hours after admission, VAS was less than 7 in only $41 \%$ of cases. The median VAS declined steadily during hospitalization. Pain intensity was not correlated with morphine dosage. Success 6 hours after admission (VAS $<7$ ) and during hospitalization (VAS£4) was associated with significantly lower VAS score at admission and lower number of VOC during the study period. Patients who experienced $>2 \mathrm{CVO} /$ year have the following characteristics: higher VAS at admission, higher morphine dosages, lower success rate and lower CRP, bilirubin, LDH and reticulocyte count.

The difficulties encountered in the management of patients who experienced $>2$ VOC/year may be related to their genotypic particularities. For such patients, an increase of morphine dosage is required. We have developed a computer routine in order to reduce time and increase accuracy of PCA prescription, and to build a prospective database that enables continuous assessment of our treatment protocol. 
1614 ANALGESIA FOR PAINFUL PROCEDURES IN CHILDREN. A SURVEY OF CURRENT PRACTICE IN SOUTH-WEST LONDON HOSPITALS

doi:10.1136/archdischild-2012-302724.1614

'D Son, ${ }^{2}$ Liu. 'Paediatrics, Queen Mary's Hospital for Children, St Helier Hospital, Carshalton, UK; ${ }^{2}$ University of Heidelberg, Heidelberg, Germany

Background and Aims Knowledge about safe and effective management of pain in children is well known and documented. There is still a gap between knowledge and everyday practice. It seems children are still getting less analgesia than adults and newborn and infants less than children.

We conducted an audit/survey to get an overview of current analgesia practice for painful procedures in cildren.

Methods We used a questionnaire, in a paper and also online format, and distributed it to nursing and midwifery staff, Paediatric and GP junior doctors, and consultants working in Paediatric departments in hospitals in the South West of London. It included general questions about the perception of pain and also specific scenarios looking into the use of analgesia for a variety of procedures in children of different ages.

Results The amount of analgesia used was highest in the age group over one year and lowest in the newborn and one to four months groups. Throughout the different age groups, venepunctures were among the procedures when analgesia was used most often. Analgesia for intramuscular injections and lumbar punctures was never or only rarely used in the groups up to 4 months. Regardless of age, the procedure most commonly performed without analgesia was intramuscular injections.

Conclusions Use and knowledge of analgesia for painful procedures in children is still not optimal. We suggest that Paediatric department should offer better training for junior staff and also include a session on this topic as part of departmental induction programmes for new staff.

\section{EFFECTS OF GLUCOSE AND NON-NUTRITIVE SUCKING ON PAIN PERCEPTION}

doi:10.1136/archdischild-2012-302724.1615

S Beken, I Hirfanoglu, K Gücüyener, E Ergenekon, Ö Turan, N Altuntaş, E Kazancı, S Ünal, F Kulalı, E Önal, C Türkyılmaz, E Koç, Y Atalay. Gazi University Hospital, Ankara, Turkey

Background and Aim Sweet tasting solutions and non-nutritive sucking are strong pain reducers for newborns. This study aimed to investigate effects of $30 \%$ glucose solution and non-nutritive sucking on amplitude of pain perception with pain scale, stress detector and near infrared spectroscopy (NIRS) during and after venipuncture.

Method Term newborns were randomised into two groups before venipuncture for bilirubin measurement.

Group 1 received 30\% dextrose,

Group 2 received sterile water before venipuncture.

Recordings of skin conductance algesimeter (SCA, Med-Storm Innovation, Norway) from foot and NIRS (NIRO 200 Hamamatsu, Japan) from head were obtained starting 7 minutes before venipuncture until 7 minutes after. PAIN scores were obtained during and 7 minutes after procedure. Non-nutritive sucking was provided throughout the study.

Results 25 patients were included. Median PAIN scores were similar in both groups during venipuncture 5 (1-8) in Group 1, and $6(1-10)$ in Group 2. Scores were decreased significantly seven minutes after procedure in both groups; as $1(0-1)$ and $3(1-3)$ in Group 1 and 2 respectively. In Group 1, cerebral blood volume (CBV) was increased from baseline after the procedure $(p=0.008)$ however in Group 2 there was a decrease in CBV from baseline without statistical significance. Cerebral blood flow (CBF) was increased from baseline in both groups without statistical significance. SCA values were slightly increased from 0.23 to 0.29 in Group 1 and from 0.23 to 0.59 in Group 2.

Conclusion Nonnutritive sucking and $30 \%$ glucose attenuate pain and stress responses during venipuncture however cerebral effects are open to investigation.

\section{PAIN EVALUATION IN PRETERM INFANTS USING SKIN CONDUCTANCE ALGESIMETER}

doi:10.1136/archdischild-2012-302724.1616

'M Ahmed, 'D Sobithadevi, 'S Mostafa, 'P Pore, 2J Maiden, ${ }^{2} \mathrm{~S}$ Boswell, 'M Molnar, ${ }^{3}$ T Reynolds. 'Paediatrics; ${ }^{2}$ Research Nurse; ${ }^{3}$ Clinical Chemistry, Burton Hospitals NHS Foundation Trust, Burton Upon Trent, UK

Background Assessment of pain is a challenge in neonatal setting. Visual, behavioural and physiological pain scales are not always reliable in premature infants. Few studies with limited sample size have been published on the reliability and efficacy of Skin Conductance Algesimeter (SCA) in monitoring pain in infants and children.

Aim To identify the clinical usefulness of SCA as a reliable and valid measure of pain intensity and stress response in preterm infants.

Methods Parents of all preterm infants admitted to the neonatal unit were invited to participate in the study. The usefulness of SCA was compared with the simultaneous measurement of 'Premature Infant Pain Profile' (PIPP) and 'Face, Legs, Activity, Cry and Consolability (FLACC) scores during invasive and/or painful procedures.

Results 46 measurements were recorded from 31 patients. The gestational age at birth ranged from $27+5$ from $35+1$. Mean PIPP scores Pre: $3.063 \pm 1.272$, Pro: 9.175 \pm 3.761 , Post 4.275 \pm 1.506 . Twotailed Paired t-test Pre - Pro, $t=10.82, \mathrm{P}<0.001$; Pre - Post, $\mathrm{t}=4.19$, $\mathrm{P}<0.001$. Mean FLACC scores Pre: $0.713 \pm 1.198$, Pro: $5.925 \pm 2.99$, Post: 0.8625 \pm 1.0919 . Two-tailed Paired t-test Pre - Pro, $t=9.51$, $\mathrm{P}<0.001$; Pre - Post, $\mathrm{t}=0.67, \mathrm{P}=0.507$. Some correlations between SCA results and PIPP/FLACC did give statistically significant correlation coefficients.

Conclusions PIPP and FLACC scores were statistically significantly increased during the procedures. Further research is needed to ascertain the usefulness of SCA in preterm infants.

\section{ADMINISTRATION OF ROPIVACAINE WITH LOW DOSE KETAMINE REDUCES CYTOKINE EXPRESSION AFTER MAJOR ABDOMINAL OPERATION IN NEWBORN WITH CONGENITAL VISCERO-ABDOMINAL DISPROPORTION}

doi:10.1136/archdischild-2012-302724.1617

D Dmytriiev. Anesthesiology and Intensive Care, Vinnitsa National Medical University, Vinnitsa, Ukraine

Background Inflammation and nociceptive sensitization are hallmarks of tissue surrounding surgical incisions. Our studies were directed towards determining if administration ropivacaine with low dose ketamine alter cytokine production after major abdominal operation in newborn with congenital viscero-abdominal disproportion.

Methods A 39 children after major abdominal operation was used to measure the effects of infiltrative administration ropivacaine $0.2 \%$ with low dose ketamine $(0.1 \mathrm{mg} / \mathrm{kg}$ i.v. $)$ administration on cytokine production in blood 45 minutes, 4 hours after operation. We examination 30 patient, undergoing major abdominal operation in children, first group receive combination ropivacaine with low dose ketamine, second group receive morphine ( $0.1 \mathrm{ml}$ per year). For statistical analysis 2 tests were used. 